\title{
The experience of falls and balance impairment for people with Charcot Marie
}

\section{Tooth disease}

\section{Abstract}

People with Charcot Marie Tooth disease (CMT) have impairments of balance and may fall more frequently than those without the condition. This qualitative study aimed to explore the experiences of falling and poor balance through focus group interviews.

Participants were recruited through local and national meetings of the CMT United Kingdom support group. Three focus groups took place, including 25 adults with CMT in total.

Thematic analysis revealed five main themes: frequent falling; cognitive burden of walking; environmental issues; external support; getting off the floor; perception of others. Participants described the physical issues of poor balance and falling, such as frequency, challenging physical environments and difficulty getting up after a fall. In addition, fear and embarrassment were discussed along with the impact on daily activities and participation.

Physical and psychological factors need to be considered when designing falls management interventions for this group.

Key words: Charcot Marie Tooth diseases; Peripheral neuropathy; Balance impairment; Falls; Fear of falling 


\section{Introduction}

Falling is a common problem for older people and those with neurological conditions (Hyndman et al., 2002; Stack and Ashburn, 1999). The consequences of falls are far reaching due to the risk of injury. Other effects include reduced mobility and avoidance of activities due to fear of falling. This can significantly impact on quality of life (Ashburn et al., 2008; Pieterse et al., 2006). A significantly higher incidence of falls has been reported for people with neuromuscular disorders (NMD) compared to healthy controls (Pieterse et al., 2006). The study questioned a mixed pathology sample of people with muscle and nerve disorders. When the subjective report of falls was compared with clinical measures, muscular weakness was implicated as a potential contributor to falls.

Charcot-Marie-Tooth disease (CMT) is the most common inherited neuromuscular disorder with an prevalence of 1 in 2500, though this varies among reported epidemiology studies between 9.7/100,000 to 82.3/100,000 (Barreto et al., 2016). People with CMT develop a slow progressive neuropathy that manifests with distal weakness and sensory loss. Increased falls have been reported in children with CMT(Burns et al., 2009) and posturography studies have highlighted the deficits in balance performance in people with CMT (Nardone et al., 2006; van der Linden et al., 2010). We found in a survey of 222 people, $89 \%$ of the 94 respondents reported falling with nearly a third stating that they fell at least once a month (Ramdharry et al. 2011).

Attention to date has focused on understanding the impact of clinical presentation on balance impairment but there has been little examination of the impact on the person. This paper explores the experience of falls and balance difficulties for people with CMT.

\section{Methods}

Design: A phenomenological approach was used for this study as this method brings together the shared, lived experiences of several individuals. This allows the description and interpretation of the phenomenon experienced (Creswell, 2007). A focus group interview was used to collect data as the group discussions and interactions can highlight the similarity of the experiences of a phenomenon (Morgan, 1997).

Ethical approval: This study was approved by the Outer North London Ethics Committee

Setting and timescale: CMT United Kingdom is a support group for people with CharcotMarie-Tooth disease in the UK. The organisation was approached for recruitment of participants to the focus groups in 2007. CMT United Kingdom has regional and national group meetings. Study information and consent forms were sent to group leaders for 
members of the group to consider participation. If there was agreement from sufficient numbers, the researcher visited the end of one of the group meetings. Members of the group were invited to stay for the focus group but were still free to withdraw at any stage, even if they had expressed initial interest.

Procedure: Group interviews of 6-10 participants were conducted, as deemed optimal methodologically (Morgan, 1997). Focus groups were tape recorded, with prior permission, and an independent note taker was also present. All focus groups were facilitated by the main researcher $(\mathrm{GR})$. The main researcher at the time of the study was a specialist neurological physiotherapist and a PhD student exploring physical functioning of people with CMT. She had clinical experience of treating people with this condition and had, at that point, collected physical measurements from people with CMT for other studies as part of her doctorate (Ramdharry et al., 2009; Ramdharry et al., 2012).

Ground rules were set before the group interview: to avoid talking over other participants; consensus and agreement did not have to be reached; that the researcher would try to obtain the views of everyone in the group. The researcher initiated the discussion with questions on how CMT limits peoples' physical function. Two open questions were posed:

1. How does CMT affect you physically?

2. How does CMT affect your ability to walk?

The researcher facilitated the discussion using focusing questions to get further information on an area, plus questions to seek clarity and confirmation.

After each group, the researcher wrote field notes and a reflective piece on the level of discussion, interpretation of the comments and how her own biases may have influenced the discussion. This was recognised during the analysis through a "bracketing" process (Ahern, 1999).

Analysis: The tapes were transcribed by an independent person who was not present at the focus groups (AT). The field notes were used to support the transcription. The two researchers (GR and AT) then independently coded the transcribed text, by highlighting and gathering significant statements, grouping them into themes and accounting for what the experiences were and how they happened (Creswell, 2007). They then came together to compare their individual results and the emerging themes were discussed, negotiated and eventually agreed.

\section{Findings}


Sample: All participants were members of the CMTUK support group. Group leaders identified three possible meetings to conduct the groups. They approached their members and invited their participation. Three focus groups of adults (over 18 years of age) with varying types of CMT were conducted with a total of twenty five people. Each group was made up of between six and ten participants. There were eight male participants and seventeen female participants of varying ages. All subjects were ambulant with or without the assistance of walking aids and/or orthoses.

The participants were open and relaxed in their discussion, often supporting and agreeing with each other's statements. It was common for them to ask questions of each other in all three focus groups. Inevitably there were quieter members of each group, but the researcher sought their opinion and there were responses from all members.

Emerging themes: Participants discussed their experience of impaired physical functioning and fatigue, that has been previously reported (Ramdharry et al., 2012). Lengthy discussions of the problems of balance and falls occurred in all three focus groups. Six themes were identified: frequent falling; cognitive burden of walking; environmental issues; external support; getting off the floor; perception of others.

Frequent falling: There was a general perception amongst participants that they fell more frequently than people who didn't have CMT.

"Yes I think you do fall more ... yes ... even when you're being careful you fall more" [Focus group 1, participant 6]

None of the respondents recalled serious injuries. They reported frequent falling when at school and in the workplace, which could impact on occupation. One person recollected:

"I used to be a nursery school teacher before I became a mum. I was tripping over all the time and falling into the children and obviously I can't do that ever again..." [Focus group 2, participant 9]

Cognitive burden of walking: In this theme, people described having to think about every step when walking to avoid trip hazards and falls. They felt that walking was not an automatic process. They needed to watch every foot fall to ensure the foot was lifted high enough to avoid tripping or be wary of obstacles.

“... normal folk just walk without thinking about it and I'm almost like, it's like conscious almost every step. It's not an automatic process, it's almost a thought about process, where you put feet... I'm always looking down at the ground too, you know, there might be a pothole in that floor out there that wasn't there earlier, you 
know, I'm going to fall down... it's not something that you just do without thinking about it, so getting up and almost a conscious process whereas I suppose most folk just get up and walk and it doesn't matter what shoes they're wearing, it doesn't matter where they're going, what surface..."

[Focus group 1, participant 4]

One person qualified that the thinking about each step was because they couldn't feel their feet. The constant observation meant that they were not able to look up at their surroundings and some felt they were missing out on seeing things around them.

"...to avoid constantly falling over I have to look at my feet and watch my feet, that they're actually coming up and down. So I can't walk along like other people do and look at the sights, because as soon as I do that, I go all over the place...." [Focus group 3, participant 1]

"...walking outside on concrete you have to look down because there are so many places you can trip, you know, even with the supports I still look down and I miss a lot, you know I've studied architecture and all sorts of things, well I can't look up at the lovely buildings because I'll end up flying." [Focus group 3, participant 3]

Environmental issues: Here participants described difficulties on uneven, rough terrain, slippery surfaces and slopes. Uneven surfaces were usually described outside, for example grass or cobblestones, but some people reported difficulties on carpet. They describe how they avoid walking on slippery surfaces e.g. in icy conditions.

“- And it's when it's wet and there's autumn leaves ...

- Yes, anything slightly slippery

- I'm really begin to lack confidence of being alone.

- Or a lot of ice on the floor and snow I mean the kids love snow but I look at it and think I'm not going out there, there might be a pothole under here!"

[Focus group 1, exchange between multiple participants] 
"Another thing I find is the floor you're stood on, if I'm stood on thick carpet I find it harder to balance [another participant adds "Yes, and grass."]. Yes, grass, um the worst things cobblestones, so on the odd occasion I've been to Covent Garden, or just out and about in the town centre, I consciously, I'm always looking at the floor anyway, looking for obstacles." [Focus group 2, participant 5]

External support: In this theme, people describe their reliance on external support, such as walking aids, orthotic devices and stable surfaces to maintain balance and prevent falls. A commonly described issue was standing for prolonged periods. People detailed their difficulty with this in social situations or when in queues. They explained their experiences of seeking out support to lean on, such as walls.

“-Yeah, it is the standing and queuing isn't it?

-I mean I could walk round the town all day but actively standing in a shop is probably what gets me more than anything.

-And if there's nothing physically there, if you haven't got a stick and there's nothing physically there to hold on to, so um in fact you get those strange like ribbon-type things and you find you're holding onto them even though they're probably not holding you very much and then l've, I've ended up in the past knocking something over and like a whole market stall fell apart thanks to me, so I find that I'm leaning on things all the time personally."

[Focus group 2, exchange between multiple participants]

In addition, they describe the benefits to balance of using walking aids and ankle braces using language implying drastic improvements:

"As far as walking outside, I can't at all unless I hold on the wall now but my walking stick's my lifeline" [Focus group 3, participant 1]

“...it was like wading through a quagmire before, you know, l'd panic, you know, l'm going to fall, will I fall over, or am I doing drop foot and everyone is staring at me? You know, having AFOs [ankle braces] for a period, I just felt in heaven and they made such a difference..." [Focus group 3, participant 3]

Getting off the floor: Participants described the ordeal of being on the floor, detailing difficulty getting up or reliance on others to assist them. This lead to concerns about causing 
harm to family members with the effort required to help them up off the floor. Some people expressed worries about falling when alone and not being able to get up independently.

“I'm slowly killing my husband... He can't pick me up any more, I'm too heavy so I have to have a chair... When I'm out and on my own, I'm always wondering what I would do if I fell in the middle of the street....I'd probably have to ring 999 [emergency services]... to get someone to pick me up." [Focus group 1, participant 1]

Perceptions of others: Many of the participants expressed concerns about how they were perceived by others. They were worried that people would think they were drunk because of their unsteady walking. They also recalled feelings of embarrassment when tripping or falling in front of other people.

“Generally people don't notice, but if you're stood somewhere where there isn't a corner to stand against or you don't have a stick with you, or whatever, then it does look really odd and I feel very self-conscious and then you see their eyes move down and like 'what's wrong with you?'” [Focus group 2, participant 5]

"I was an usher at a friend's wedding... and of course they've got me on the wedding video tripping over down the aisle...I didn't go flat on my face, but I nearly did and of course, it's on the video." [Focus group 3, participant 4]

\section{Discussion}

This is the first qualitative study of the problem of falls and balance impairment for people with CMT. They expressed the reality of living with poor balance, highlighting the challenges and impact of this physically and psychologically.

Increased frequency of falls compared to people without CMT appeared to be the perception of participants. This is supported by our survey where $89 \%$ of 94 respondents reported falling at least once a year (Ramdharry et al., 2011). The survey also found It is surprising that more serious injuries were not discussed in the focus groups. The same survey found only $2.8 \%$ of reported falls events led to injuries requiring medical intervention (Ramdharry et al., 2011). This is a lifelong, slowly progressing condition that can start in childhood. It is possible that people have learned to fall and minimise injury, but there is no evidence to date to support this hypothesis. It should also be considered that this will have been a relatively young sample and this anomaly of frequent falls with few injuries has also been observed in 
multiple sclerosis, another neurological condition that has a relatively young population (Gunn et al., 2014).

The theme of the cognitive burden of avoiding hazards may relate to sensory compensation that occurs where there is proprioceptive loss. A laboratory based study has made the connection between reliance on vision and postural stability in people with CMT type 1A (van der Linden et al., 2010), though this was in quiet standing rather than walking. Participants of the focus groups seem to rely on visual feedback, describing watching their feet. This requires increased attention so decreasing the automatic nature of posture and balance. This is a common experience of people with other neurological disorders (Marchese et al., 2003; Wang et al., 2015). This could leave people vulnerable to falls when distracted or when in busy outdoor settings. Further exploration of the cost of a dual task condition on postural stability could ascertain the magnitude of the effect and whether people would benefit from balance training that specifically targets this (Wang et al., 2015).

Participants also described challenges with uneven or slippery surfaces. This may relate to a person's reaction to an unexpected postural perturbation from a slip, trip or an ankle giving way. A rapid reaction would be required to correct for the perturbation and prevent a fall. This requires intact high frequency sensory feedback and/or sufficient force production capacity of the ankle muscles. Slowed responses to perturbations while walking have been observed in people with CMT type $1 \mathrm{~A}$ that correlated with 1 a afferent sensory function (vibration threshold) and muscle strength (van der Linden et al., 2009). In addition, participants mentioned how balance is challenged on compliant surfaces such as carpet. A study of people with diabetic neuropathy showed reduced postural stability on foam as the softer surface alters sensory feedback (Dickstein et al., 2001). This effect was worse when eyes were closed.

The need for external support was highlighted by participants, in the form of walking aids and orthoses. The use of walking aids in this group has not been explored, but studies in other forms of neuropathy imply that light touch feedback to the hand from a walking stick could improve postural stability (Dickstein et al., 2001). The use of ankle foot orthoses, however, has been studied with positive effects such as reduced foot drop, improved energy expenditure and balance (Ramdharry et al., 2012). There can be varying attitudes to the use of orthoses, however, with some suggestion of self-selection according to comfort, cosmesis and level of disease severity (Ramdharry et al., 2012).

Participants described difficulties getting off the floor once they had fallen. There were fears expressed about getting stranded and having to rely on either family members or the emergency services for rescue. There is a potential impact of avoiding walking outdoors for 
fear of falling that can have a knock on effect on overall physical activity. In addition, the higher cognitive burden identified could also contribute to avoidance of outdoor walking due to higher effort and greater risk of distraction. Studies of pedometry have shown reduced walking volumes (Ramdharry et al., 2016) and step climbing (Menotti et al., 2014) in people with CMT, though the reasons were not explored and could be due to other factors such as fatigue.

In addition to worries about getting stranded, fear was also expressed in the context of safety at work and judgements of others. There were concerns of negative labelling, particularly with regards to being drunk, with feelings of embarrassment if falls or stumbles were observed. Concerns about the opinions of others were also echoed in regard to the impact of fatigue, as previously reported (Ramdharry et al., 2012b).

There were key limitations of this study. People with CMT are not a homogenous group with widely varying degrees of severity. Focus groups can highlight the sharing of experiences but due to the small number of groups, there may not have been discussion of a sufficient range of issues. All people who attended the groups were ambulant adults, and were also members of the CMT United Kingdom support group. This will have created a sampling bias, as the participants may have already reached a degree of acceptance of their condition by being active members of their local groups. It is possible that people with milder disease are less likely to become members so would have been under represented, along with very severely affected, non-ambulant people.

\section{Future work}

We are currently undertaking a laboratory based study to understand the correlates of presenting impairments and falls frequency. We are also exploring use of walking aids, and conducting a pilot study of home based balance training for people with CMT 1A. We hope the new knowledge from this body of work will provide future guidance on management of falls and balance impairment encountered by people with CMT.

\section{Conclusion}

This is the first qualitative study to explore the problem of falls and poor balance experienced by people with CMT. The aim of this study was to hear their descriptions of living with this phenomenon. The focus groups revealed the physical impact of sensorimotor impairment on postural stability and recovery, but fear and embarrassment were expressed in response to the challenges that balance impairment present. Impact and restriction of daily activity and 
occupation are consequences that need considering when supporting people with CMT to manage falls.

\section{Key points:}

1. People with CMT perceive that they fall more frequently than people without the condition

2. They report having to concentrate on every step, which could leave them vulnerable to distraction or situations of divided attention

3. Their physical limitations lead to difficulties walking on uneven surfaces and requiring external support

4. There is a fear of being stranded on the floor and concern for family members who have to help them get up

5. They are embarrassed if they are unsteady in public and are concerned that other people will interpret their imbalance as being due to excessive alcohol consumption

\section{Acknowledgements}

This work was funded by the National Institute for Health Research, the Medical Research Council and the Charcot-Marie-Tooth United Kingdom Charity (www.cmt.org.uk). MMR is grateful to the Medical Research Council (MRC), MRC Centre grant (G0601943), and the National Institutes of Neurological Diseases and Stroke and office of Rare Diseases (U54NS065712) for their support. The INC (U54NS065712) is a part of the NCATS Rare Diseases Clinical Research Network (RDCRN). We are also supported by the National Institute for Health Research (NIHR) University College London Hospitals (UCLH) Biomedical Research Centre (BRC).

\section{All of the authors of this manuscript report no conflicts of interest.}

\section{References:}

Ahern, K.J., 1999. Ten Tips for Reflexive Bracketing. Qual. Health Res. 9, 407-411. doi:10.1177/104973239900900309

Ashburn, A., Stack, E., Ballinger, C., Fazakarley, L., Fitton, C., 2008. The circumstances of falls among people with Parkinson's disease and the use of Falls Diaries to facilitate reporting. Disabil. Rehabil. 30, 1205-1212. doi:10.1080/09638280701828930 
Barreto, L.C.L.S., Oliveira, F.S., Nunes, P.S., de França Costa, I.M.P., Garcez, C.A., Goes, G.M., Neves, E.L.A., de Souza Siqueira Quintans, J., de Souza Araújo, A.A., 2016. Epidemiologic Study of Charcot-Marie-Tooth Disease: A Systematic Review. Neuroepidemiology 46, 157-165. doi:10.1159/000443706

Burns, J., Ryan, M.M., Ouvrier, R.A., 2009. Evolution of foot and ankle manifestations in children with CMT1A. Muscle Nerve 39, 158-166. doi:10.1002/mus.21140

Creswell, J.W., 2007. Qualitative Inquiry and Research Design: Choosing Among Five Approaches, Second Edition. ed. Sage Publications, Inc.

Dickstein, R., Shupert, C.L., Horak, F.B., 2001. Fingertip touch improves postural stability in patients with peripheral neuropathy. Gait Posture 14, 238-247. doi:10.1016/S09666362(01)00161-8

Dufek, J.S., Neumann, E.S., Hawkins, M.C., O'Toole, B., n.d. Functional and dynamic response characteristics of a custom composite ankle foot orthosis for CharcotMarie-Tooth patients. Gait Posture. doi:10.1016/j.gaitpost.2013.07.121

Gunn, H., Creanor, S., Haas, B., Marsden, J., Freeman, J., 2014. Frequency, characteristics, and consequences of falls in multiple sclerosis: findings from a cohort study. Arch. Phys. Med. Rehabil. 95, 538-545. doi:10.1016/j.apmr.2013.08.244

Hachisuka, K., Ohnishi, A., Yamaga, M., Dozono, K., Ueta, M., Ogata, H., 1997. The role of weakness of triceps surae muscles in astasia without abasia. J. Neurol. Neurosurg. Psychiatry 62, 496-500.

Hyndman, D., Ashburn, A., Stack, E., 2002. Fall events among people with stroke living in the community: circumstances of falls and characteristics of fallers. Arch. Phys. Med. Rehabil. 83, 165-170.

Marchese, R., Bove, M., Abbruzzese, G., 2003. Effect of cognitive and motor tasks on postural stability in Parkinson's disease: A posturographic study. Mov. Disord. 18, 652-658. doi:10.1002/mds. 10418

Menotti, F., Laudani, L., Damiani, A., Macaluso, A., 2014. Amount and intensity of daily living activities in Charcot-Marie-Tooth 1 A patients. Brain Behav. 4, 14-20. doi:10.1002/brb3.187

Menotti, F., Laudani, L., Damiani, A., Mignogna, T., Macaluso, A., 2013. An anterior anklefoot orthosis improves walking economy in Charcot-Marie-Tooth type $1 \mathrm{~A}$ patients. Prosthet. Orthot. Int. 0309364613506250. doi:10.1177/0309364613506250

Morgan, D., 1997. Focus Groups as Qualitative Research, 2nd Revised edition. ed. Sage Publications, Inc.

Nardone, A., Grasso, M., Schieppati, M., 2006. Balance control in peripheral neuropathy: are patients equally unstable under static and dynamic conditions? Gait Posture 23, 364373. doi:10.1016/j.gaitpost.2005.04.002

Pieterse, A.J., Luttikhold, T.B., de Laat, K., Bloem, B.R., van Engelen, B.G., Munneke, M., 2006. Falls in patients with neuromuscular disorders. J. Neurol. Sci. 251, 87-90. doi:10.1016/j.jns.2006.09.008

Ramdharry, Gita M., Day, B.L., Reilly, M.M., Marsden, J.F., 2012. Foot drop splints improve proximal as well as distal leg control during gait in Charcot-Marie-Tooth Disease. Muscle Nerve 46, 512-519. doi:10.1002/mus.23348

Ramdharry, G.M., Day, B.L., Reilly, M.M., Marsden, J.F., 2009. Hip flexor fatigue limits walking in Charcot-Marie-Tooth disease. Muscle Nerve 40, 103-111. doi:10.1002/mus.21264

Ramdharry, G.M., Entwistle, L., Reilly, M.M., 2011. Falls in adults with Charcot-Marie-Tooth disease. J. Peripher. Nerv. Syst. 16, S115.

Ramdharry, G.M., Pollard, A.J., Grant, R., Dewar, E.L., Laurá, M., Moore, S.A., Hallsworth, K., Ploetz, T., Trenell, M.I., Reilly, M.M., 2016. A study of physical activity comparing people with Charcot-Marie-Tooth disease to normal control subjects. Disabil. Rehabil. 1-6. doi:10.1080/09638288.2016.1211180

Ramdharry, Gita M, Thornhill, A., Mein, G., Reilly, M.M., Marsden, J.F., 2012. Exploring the experience of fatigue in people with Charcot-Marie-Tooth disease. Neuromuscul. Disord. NMD 22 Suppl 3, S208-213. doi:10.1016/j.nmd.2012.10.016 
Stack, E., Ashburn, A., 1999. Fall events described by people with Parkinson's disease: implications for clinical interviewing and the research agenda. Physiother. Res. Int. J. Res. Clin. Phys. Ther. 4, 190-200.

van der Linden, M.H., de Kam, D., van Engelen, B.G.M., Hendricks, H.T., Duysens, J., 2009. Fast responses to stepping on an unexpected surface height depend on intact largediameter nerve fibers: a study on Charcot-Marie-Tooth type $1 \mathrm{~A}$ disease. J. Neurophysiol. 102, 1684-1698. doi:10.1152/jn.91142.2008

van der Linden, M.H., van der Linden, S.C., Hendricks, H.T., van Engelen, B.G.M., Geurts, A.C.H., 2010. Postural instability in Charcot-Marie-Tooth type $1 \mathrm{~A}$ patients is strongly associated with reduced somatosensation. Gait Posture 31, 483-488. doi:10.1016/j.gaitpost.2010.02.005

Wang, X.-Q., Pi, Y.-L., Chen, B.-L., Chen, P.-J., Liu, Y., Wang, R., Li, X., Waddington, G., 2015. Cognitive motor interference for gait and balance in stroke: a systematic review and meta-analysis. Eur. J. Neurol. 22, 555-e37. doi:10.1111/ene.12616 